\section{Percutaneous treatment of severe retroperitoneal hematoma after percutaneous coronary intervention}

\author{
Agarwal Rajendra Kumar ${ }^{1 *}$ and Agarwal Rajiv ${ }^{2}$ \\ 'Senior Consultant, Cardiology, Max Smart Super Speciality Hospital, Saket, New Delhi, India \\ ${ }^{2}$ Senior Director, Cardiology, Max Smart Super Speciality Hospital, Saket, New Delhi, India
}

\section{Abstract}

We describe a patient who developed severe retroperitoneal and intraperitoneal bleeding complicating femoral arterial catheterization for Percutaneous coronary intervention. Balloon tamponade of the actively bleeding femoral artery was effective in sealing off the leakage.

This management strategy for this problem emphasizing an anatomical based interventional approach if the patient does not stabilize with volume resuscitation.

\section{Introduction}

Retroperitoneal bleeding is an uncommon but potentially fatal complication of cardiac catheterization performed using the transfemoral route. While it is usually self-limiting but bleeding can be severe and fatal especially when the patient is anticoagulated [1]. Usually surgical exploration is needed but due to hypocoagulability, may not be effective in this group of patients [2]. We report our experience of successful percutaneous management of severe retroperitoneal bleeding complicating catheterization.

\section{Case report}

A 51-year-old male, nondiabetic with a history of angina on exertion and strongly positive exercise stress test for reversible myocardial ischemia with normal LV systolic function underwent left heart cardiac catheterization. He was found to have significant coronary artery disease (LAD/ D1 true bifurcation lesion and significant stenosis in the proximal to mid circumflex artery. A $2.5 \times 33 \mathrm{~mm}$ Xience stent (Abbott Vascular) was deployed in Left Circumflex artery. PCI to LAD/D1 true bifurcation was done with DK crush technique with a $3 \times 38 \mathrm{~mm}$ Xience stent (Abbott Vascular) in LAD and 2.5x $18 \mathrm{~mm}$ Xience stent (Abbott Vascular) in D1 with good angiographic results. Aspirin and Ticagrelor were used as an antiplatelets. Intravenous antiplatelet were not given. Right common femoral arterial access was used which was a difficult puncture with three attempts. Right femoral venous access was also taken (single attempt). The patient

\begin{abstract}
More Information
*Address for Correspondence: Agarwal Rajendra Kumar, Senior Consultant, Cardiac Intervention and Electrophysiology, MD (Medicine), DNB (Cardiology), FNB (Interventional Cardiology), Fellowship-Cardiac Pacing and Electrophysiology, Advanced EP Training- Liverpool, Member of EHRA, UK, Tel: +91 9560919636;

Email: drrajendra2106@gmail.com

Submitted: May 10, 2021

Approved: September 24, 2021

Published: September 25, 2021

How to cite this article: Kumar AR, Rajiv A. Percutaneous treatment of severe retroperitoneal hematoma after percutaneous coronary intervention. J Cardiol Cardiovasc Med. 2021; 6: 055-058.
\end{abstract}

DOI: 10.29328/journal.jccm.1001119

Copyright: @ 2021 Kumar AR, et al. This is an open access article distributed under the Creative Commons Attribution License, which permits unrestricted use, distribution, and reproduction in any medium, provided the original work is properly cited.

Keywords: Catheterization; Femoral artery access complications; Retroperitoneal hemorrhage/bleeding; Balloon tamponade

Check for updates

( $)$ OPEN ACCESS

was sent back to the coronary care unit with the instruction of removal of both femoral sheaths when activated clotting time (ACT) is less than 180 seconds and to be followed by manual compression and a tight pressure bandage to prevent hematoma. Thirty minutes after the procedure, the patient became hypotensive (systolic BP $70 \mathrm{mmHg}$ ) with bradycardia (heart rate: 36 beats/min) and treated as vasovagal syncope with atropine and Intravenous fluid. There was continuous blood oozing from sides of arterial sheath and from another arterial puncture site which was not responding to manual compression so both sheaths were removed in order to have more effective manual compression. Total one-hour manual compression given which led to no ooze in right groin even on coughing and then a compression bandage done. He have two more episodes of hypotension in next one hour in spite of rapid intravenous fluid transfusion. So inotropes with noradrenaline infusion was also initiated but blood pressure remained borderline. He was feeling fatigue and dizzy. Reinspection of right groin showed hemorrhagic bluish discoloration with scrotal swelling. The hemoglobin concentration fell from 15.1 (preprocedure Lab value) to 10.5 (ABG value) grams/ 
deciliter. A CT angio scan of the abdomen and groin arteries showed a retroperitoneal and intraperitoneal hematoma with extravasation of contrast from right common femoral artery in to surrounding soft tissue suggestive of active leak of contrast, extending in the subcutaneous tissue in groin and lower right inguinal region (Figure 1). The patient was transferred back to the cardiac Cath lab because of persistent hypotension despite fluid resuscitation. Left common femoral arterial access was obtained and a 6 French sheath was introduced. A Judkins right (JR6) diagnostic catheter was passed retrogradely into the left common iliac artery and then antegradely into the right iliofemoral arterial system over a 0.035 inch guide wire. Digital subtraction angiography (DSA) showed the location of the retroperitoneal hematoma (RPH) (Figure 2).

Through the Judkins right (JR) 6 catheter, a 0.035 inch magic torque guide wire $(260 \mathrm{~cm})$ [Boston Scientific] was passed across the common femoral artery into the distal right superficial femoral artery. The JR6 catheter was then withdrawn leaving the wire in the distal superficial femoral artery. A 8 x 40 Armada 35 PTA Balloon (Abbott Vascular) was inserted over the magic torque guide wire and balloon tamponade was performed over the leaking site for a total of $15 \mathrm{~min}$ at a inflation pressure of 4 atm (Figure 3). Total two inflations were done with a gap of 5 minute in between.

Post balloon tamponade angiogram revealed a complete sealing of the leakage with no further extravasation of contrast (Figure 4).

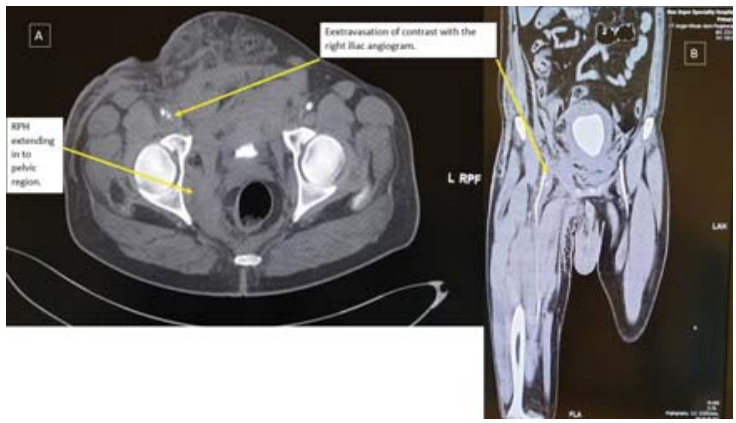

Figure 1: Contrast computerized tomography of a retroperitoneal hemorrhage are shown in (A) coronal and (B) sagittal views. A large amount of extravascular contrast with the right iliac angiogram location near the middle third of the femoral head, suggestive of active bleeding from mid right common femoral artery into the pelvis.

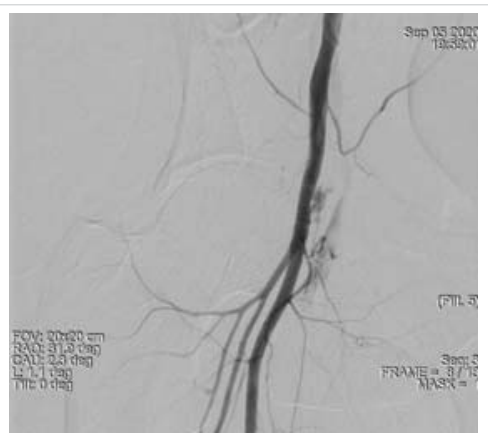

Figure 2: Demonstrates a large amount of extravascular contrast with the right iliac angiogram location near the middle third of the femoral head. suggestive of active bleeding from mid right common femoral artery into the pelvis.

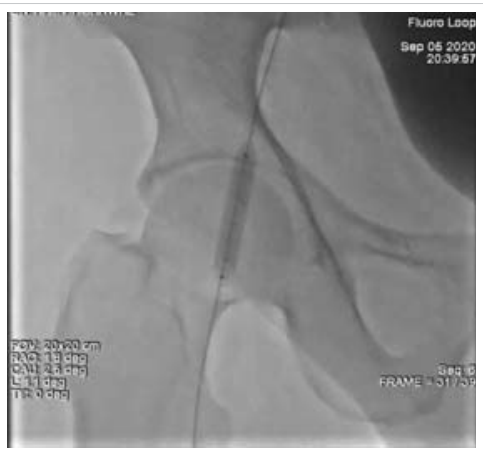

Figure 3: Demonstrates Balloon tamponade of the leaking right common femoral artery via left femoral approach.

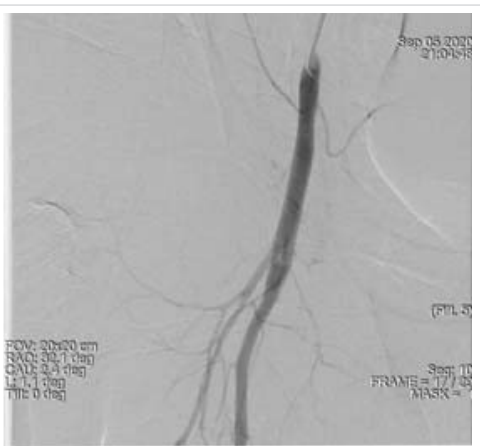

Figure 4: Post balloon tamponade angiogram indicating complete sealing of the leakage.

The patient was sent back to the coronary care unit with the instruction of blood transfusion and gradual tapering of noradrenaline. Patient was rapidly stabilized and there was no sign of rebleeding afterward. Two units of packed red blood cells were transfused. The patient was hospitalized for 5 days and was fully ambulatory at the time of discharge.

\section{Discussion}

Bleeding after transfemoral catheterization is a wellrecognized complication and significant hematomata may occur in the groin and retroperitoneal spaces.

Female gender, low body surface area (BSA), higher femoral artery puncture, chronic renal insufficiency (creatinine $>2$ milli-grams/deciliter), Glycoprotein IIb/IIIa inhibitors infusion, body surface area $<1.8 \mathrm{~m}^{2}$ are significant risk factors for RPH.

A higher femoral arterial puncture site is defined as a puncture above the inguinal ligament or above the middle $1 / 3$ of the femoral head on fluoroscopy or above the inferior epigastric artery (IEA) on angiography IEA is a surrogate angiographic marker for the inguinal ligament and the retroperitonium [2-4]. However, a lower arterial puncture does not completely eliminate the risk of RPH and can be explained by direct superior connection of the anatomical femoral sheath with the retroperitoneum allowing bleeding from the femoral vascular structures contained within the femoral sheath to reach the retroperitoneum [3]. 
Computed tomography (CT) study for these hematomas identifies four different anatomic locations relating to different routes of bleeding. These typical locations are retroperitoneal, intraperitoneal, groin and thigh and abdominal wall. The hematoma type so formed probably depends on whether the bleeding is confined to the femoral sheath and/or spreads into the femoral triangle as bleeding spreads along fascial planes [5].

The case presented here can be considered as a case of RPH resulting from normal lower site puncture of common femoral artery as contrast leakage on angiography is at the level of the middle $1 / 3$ of the femoral head only (Figure 5). Here RPH can be due to multiple puncture (two leakage points can be seen on angiography) and can be explained by direct superior connection of the anatomical femoral sheath with the retroperitoneum leading to RPH [3]. The other risk factor could be a low BSA which was 1.61 meter $^{2}$.

\section{Management}

There are no randomized trials to guide the treatment strategies for RPH and the evidence is based on small cohort series or isolated case reports.

Surgical intervention is only indicated in a small portion of these patients as most of these cases of retroperitoneal hematomata can be managed by blood transfusion and close hemodynamic monitoring alone as most are self-limiting. If a patient remains unstable in spite of aggressive resuscitation, we believe the preferred strategy is an initial percutanaeous approach while open surgery should be used only if the bleeding cannot be controlled [6].

Few studies have reported on interventional treatment in patients with spontaneous RPH with use of coils, gelatin and/ or polyvinyl alcohol for embolization [7]. Depending on the vessel size either local thrombin injection or if the vessel is large enough then microcoil emolization can be undertaken.

When the bleeding site is in the external iliac artery above the femoral head then a covered stent can be placed taking care to avoid a bending point.

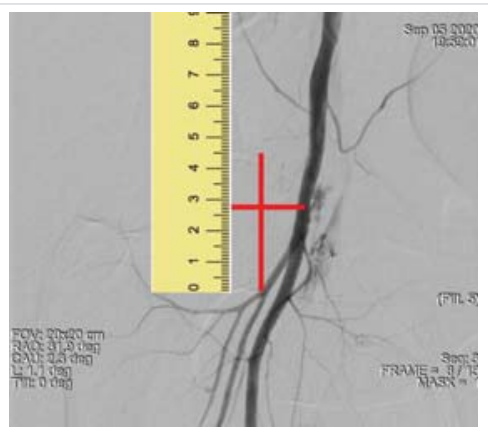

Figure 5: Demonstrates a large amount of extravascular contrast with the right iliac angiogram location at the level of middle third of the femoral head with two leakage points. suggestive of active bleeding from mid right common femoral artery into the pelvis.
In a patient with massive RPH who had undergone diagnostic cardiac catheterization, Mak, et al. described balloon tamponade of the common femoral artery [8]. Samal and White have also described percutaneous approaches for the management of access site complications [9]. Prolonged balloon inflations has also been found effective for management of coronary artery perforation while patient undergoing for percutaneous intervention. [10].

Based on 2 case reports a clinical Algorithm for Retroperitoneal Hemorrhage has also been described (6). The approach describes with initial angiography to identify the exact bleeding site will dictate the best option for treatment.

In our case, a patient of Post PCI retroperitoneal hemorrhage suffering from hypovolemic shock with massive groin, retroperitoneal, and intraperitoneal hematomata was successfully treated by percutaneous transluminal balloon tamponade (PTBT). This technique is rapidly instituted in a hemodynamically unstable patient and allows time to treat and improve the hemodynamics in a controlled environment and to plan a careful strategy to treat the bleeding vessel based on the anatomy producing the bleeding. The equipment needed to perform this procedure is present in a standard cardiac cath lab that does PCIs. This approach of percutaneous transluminal balloon tamponade (PTBT) allows time to treat and improve the hemodynamics in a controlled environment and to plan a careful strategy based on the bleeding producing anatomy. The platform (6 or 7 French sheath) needed here allows for the delivery of a covered stent if the bleeding does not seal by the balloon tamponade. Likewise if there has been disruption of the IEA this technique allows time to plan a coil emolization or thrombin injection strategy if needed. Vascular surgical consultation can be entertained should this conservative approach not be successful.

\section{Conclusion}

Percutaneous transluminal balloon tamponade should be considered as an effective therapeutic option for the management of uncontrolled arterial entry site producing the RPH following cardiac catheterization.

\section{References}

1. Donglas JS, King SB. Management of complications. In King SB, Donglas JS (eds): "Coronary Arteriography and Angioplasty." New York: McGraw-Hill, Inc. 1985; 311.

2. Pereira BJ, Ramprasad KS. Retroperitoneal hemorrhage following trauma during femoral vein cannulation for Haemodialysis: A therapeutic dilemma. Ren Fail. 1989; 11: 221-222. PubMed: https://pubmed.ncbi.nlm.nih.gov/2485486/

3. Farouque HMO, Tremmel JA, Raissi Shabari F. Risk factors for the development of retroperitoneal hematoma after percutaneous coronary intervention in the era of glycoprotein Ilb/IIla inhibitors and vascular closure devices. J Am Coll Cardiol. 2005; 45: 363-368.

PubMed: https://pubmed.ncbi.nlm.nih.gov/15680713/

4. Sherev DA, Shaw RE, Brent BN. Angiographic predictors of femoral access site complications: Implication for planned percutaneous 
coronary intervention. Catheter Cardiovasc Interv. 2005; 65: 196-202. PubMed: https://pubmed.ncbi.nlm.nih.gov/15895402/

5. Trerotola SO, Kuhlman JE, Fishman EK, Russell H. CT and anatomic study of post catheterization hematomas. Radiographics. 1991; 11 247-258.

PubMed: https://pubmed.ncbi.nlm.nih.gov/1827528/

6. Sajnani N, Bogart DB. Retroperitoneal Hemorrhage as a Complication of Percutaneous Intervention: Report of 2 Cases and Review of the Literature. Open Cardiovasc Med J. 2013; 7: 16-22.

PubMed: https://www.ncbi.nlm.nih.gov/pmc/articles/PMC3617546/

7. Pathi R, Voyvodic F, Thompson WR. Spontaneous extraperitoneal haemorrhage: computed tomography diagnosis and treatment by selective arterial embolization. Australas Radiol. 2004; 48: 123-128. PubMed: https://pubmed.ncbi.nlm.nih.gov/15230743/

8. Mak GYK, Daly B, Chan W. Percutaneous treatment of post catheterization massive retroperitoneal hemorrhage. Cathet Cardiovasc Diagn. 1993; 29: 40-43.

PubMed: https://pubmed.ncbi.nlm.nih.gov/8495470/

9. Samal AK, White CJ. Percutaneous management of access site complications. Catheter Cardiovasc Interv. 2002; 57: 12-23.

PubMed: https://pubmed.ncbi.nlm.nih.gov/12203921/

10. Meguro $\mathrm{K}$, Ohira $\mathrm{H}$. Outcome of prolonged balloon inflation for the management of coronary perforation. J Cardiol. 2013; 61: 206-209. PubMed: https://pubmed.ncbi.nlm.nih.gov/23380534/ 